\title{
Lead exposure in preterm infants receiving red blood cell transfusions
}

\author{
Hijab Zubairi' , Paul Visintainer ${ }^{2,3}$, Jennie Fleming ${ }^{1}$, Matthew Richardson ${ }^{1,3}$ and Rachana Singh ${ }^{1,3}$
}

BACKGROUND: Preterm infants may inadvertently be exposed to lead from the packed red blood cell (pRBC) transfusions with almost no or very limited data available. The aim of the study was to quantify this exposure in preterm infants $\leq 30$ wk gestational age (GA).

METHODS: Prospective cohort study, infants $\leq 30$ wk GA were eligible, infants $<23$ wk GA and known chromosomal diseases were excluded. Blood lead levels (BLLs) were obtained at birth, before and after each transfusion, and at discharge. BLLs were also obtained from the donor pRBC aliquot transfused. A linear mixed model analysis was done.

RESULTS: Of 75 infants, 34 received a total of 126 pRBC transfusions. Each infant had an average of 3.7 transfusions. $92 \%$ of lead levels in the transfused aliquot were $\leq 5 \mathrm{mcg} / \mathrm{dl}$, $6.8 \%$ were between $6-8 \mathrm{mcg} / \mathrm{dl}$ and 1 had a level of $56 \mathrm{mcg} /$ dl. Average total lead load was $1.3 \mathrm{mcg} / \mathrm{dl}$. For each $1 \mathrm{mcg} / \mathrm{dl}$ increase in transfused pRBC lead level, infant's post-transfusion BLL increased by $0.20 \mathrm{mcg} / \mathrm{dl}(95 \% \mathrm{Cl}: 0.07 \mathrm{mcg} / \mathrm{dl}, 0.33 \mathrm{mcg} /$ $\left.\mathrm{dl}_{;} P=0.002\right)$, adjusting for $\mathrm{GA}$ and birth weight. There was no significant increase in discharge BLLs, which were similar for both transfused and nontransfused infants.

CONCLUSION: Post-transfusion infant BLLs correlate significantly with the transfused pRBCs lead level.

ead is a known potent neurotoxicant with effects on cognition, behavior, and intelligence quotient (IQ) (1). The Centers for Disease Control and Prevention (CDC) and the American Academy of Pediatrics recommend screening all children for lead toxicity starting at age one. Current data show that even low lead levels may have detrimental effects and as such there is no safe lead level (2). Based on these findings, current CDC guidelines have altered the reference value for lead toxicity from 10 to $5 \mathrm{mcg} / \mathrm{dl}(2,3)$.

Several studies have shown a relationship between elevation in lead levels and decrease in IQ (4-7). Lanphear et al. (8) observed in their pooled analysis that the lead-associated IQ deficits were significantly greater at lower blood lead concentrations $(<7.5 \mathrm{mcg} / \mathrm{dl})$. The developing brain of a preterm infant is more vulnerable to the adverse effects of lead exposure as compared to term infants and children; especially since there is growing evidence that there may be no safe lead level (9-11) Even trace amounts of lead exposure in animal models have been shown to impact optimal neurite growth (12). Additionally, preterm infants are also at increased risk for anemia of prematurity and may receive multiple packed red blood cell (pRBC) transfusions during their stay in the neonatal intensive care units with wide variations in guidelines between neonatal intensive care units (13-16). The pRBC transfusions may thus potentially increase delivery of lead to the preterm infants from the donor $\mathrm{pRBC}$ aliquots.

In this study, we hypothesized, that preterm infants receiving multiple pRBC transfusions are at risk for high lead exposure which in turn could be associated with increased posttransfusion BLL. Our main objective was to quantify the impact of $\mathrm{pRBC}$ transfusions on the lead levels of preterm infants.

\section{RESULTS}

During the study period 140 infants born met the eligibility criteria, of these 65 infants were not enrolled either due to receiving transfusions $(n=9)$ or death $(n=4)$ prior to consenting; out born delivery missing baseline samples $(n=5)$ or parental refusal to participate in the study $(n=47)$. A total of 75 very-low-birth-weight infants with a mean gestational age of 27.6 ( $\mathrm{SD} \pm 2.4$ ) wk and mean birth weight of 1,053 (SD $\pm 326) \mathrm{g}$ were consented and enrolled in the study cohort. Table 1 shows the baseline characteristics of the entire cohort as well as comparative data for the transfused vs. nontransfused infants. There were 126 transfusions among 34 infants ( $45 \%$ of the cohort). Transfused infants received an average of 3.7 transfusions (ranging from 1-8). $92 \%$ of the lead levels in the transfused aliquot were $\leq 5 \mathrm{mcg} / \mathrm{dl}, 7 \%$ had a level between 6-8 mcg/dl, and one had a level of $56 \mathrm{mcg} / \mathrm{dl}$. This data was considered as an outlier and not included for data analysis. The average total lead load per transfusion was $1.3 \mathrm{mcg}$ with a range of $0-8.6 \mathrm{mcg}$.

The multilevel regression analysis was based on 95 transfusions and 33 infants that had complete data for pre- and posttransfusion infant BLL, transfused pRBC aliquot lead level, gestational age, and birth weight. Regression results showed for each $1 \mathrm{mcg} / \mathrm{dl}$ increase in transfused $\mathrm{pRBC}$ lead level, infant post-transfusion BLL increased by $0.20 \mathrm{mcg} / \mathrm{dl}$ (95\% CI: 0.07 
Table 1. Distribution of clinical characteristics, overall and by transfusion group

\begin{tabular}{|c|c|c|c|c|}
\hline & Overall $(n=75)$ & No transfusion $(n=41)$ & Any transfusion $(n=34)$ & \\
\hline Characteristics & Mean (SD) & Mean (SD) & Mean (SD) & $P$ \\
\hline Gestational age, wk; median (range) & $27.6(2.4) ; 28.3(23.0,30.9)$ & $29.1(1.6) ; 29.4(24.0,30.9)$ & $25.8(1.8), 25.3(23.0,29.4)$ & $<0.001$ \\
\hline Admission HCT; median (range) & $0.44(0.06) ; 0.45(0.28,0.71)$ & $0.47(0.06) ; 0.46(0.37,0.71)$ & $0.42(0.05) ; 0.43(0.28,0.48)$ & $<0.001$ \\
\hline Discharge $\mathrm{HCT}$; median (range) & $0.29(0.04) ; 0.29(0.20,0.39)$ & $0.29(0.03) ; 0.29(0.23,0.36)$ & $0.30(0.04) ; 0.29(0.20,0.39)$ & 0.06 \\
\hline \multirow[t]{2}{*}{ Cord blood lead, mcg/dla; median (range) } & $0.50(0,2)$ & $1(0,2)$ & $0.50(0,2)$ & $0.41^{\mathrm{b}}$ \\
\hline & $\%(n)$ & $\%(n)$ & $\%(n)$ & \\
\hline Male & $65.3(49)$ & $61.0(25)$ & $70.6(24)$ & 0.47 \\
\hline C-section & $50.7(38)$ & $46.3(19)$ & $55.9(19)$ & 0.49 \\
\hline Severe IVH $(\mathrm{G} 3, \mathrm{G} 4)$ & $9.3(7)$ & $0.0(0)$ & $20.6(7)$ & 0.003 \\
\hline
\end{tabular}

${ }^{a} n=71 ; 39$ no transfusion, 32 any transfusion. bWilcoxon rank-sum test.

$\mathrm{HCT}$, hematocrit; IVH, intraventricular hemorrhage; $P V L$, periventricular leukomalacia.

$\mathrm{mcg} / \mathrm{dL}, 0.33 \mathrm{mcg} / \mathrm{dl} ; P=0.002$ ), adjusting for gestational age, birth weight, and pretransfusion BLL. The model $R^{2}$ was 0.29 for the model with transfused $\mathrm{pRBC}$ lead level as a continuous variable and 0.37 for transfused pRBC lead level modeled categorically with indicator variables (Figure 1). Once the transfusion pRBC lead level and the pretreatment lead level were included in any model, no patient characteristic achieved a $P$ value of less than 0.30 . These characteristics included sex, delivery type, presence of severe lesion, admission hematocrit, and breast feeding.

Of the 70 infants with discharge BLLs, 57\% $(n=40)$ had discharge BLLs of 0 and the highest BLL was $2 \mathrm{mcg} / \mathrm{dl}$. A comparison of infants who were transfused $(n=32)$ and those who were not $(n=38)$, noted no difference for discharge BLL. For both groups, the maximum discharge BLL was $2 \mathrm{mcg} / \mathrm{dl}$. The mean discharge BLL was $0.4 \mu \mathrm{g} / \mathrm{dl} \pm 0.6$ in the transfused group vs. $0.4 \mu \mathrm{g} / \mathrm{dl} \pm 0.6$ in the nontransfused group $(P=0.91)$.

One transfused aliquot had a lead level of $56 \mathrm{mcg} / \mathrm{dl}$ and only one infant received a single transfusion from this aliquot with a total lead load of $7.84 \mathrm{mcg}$ from the single transfusion. This infant's pretransfusion lead level was $<1 \mathrm{mcg} / \mathrm{dl}$ with the post-transfusion lead level increasing to $9 \mathrm{mcg} / \mathrm{dl}$. The infant's discharge lead level was $1 \mathrm{mcg} / \mathrm{dl}$. This aliquot was not used for any further transfusions and no other infants were exposed to this elevated lead level.

\section{DISCUSSION}

Lead toxicity continues to be an important public health concern for all children in the United States (2) Currently, approximately half a million children have a lead level $>5 \mathrm{mcg} /$ $\mathrm{dl}$ in the United States with a potential for detrimental neurodevelopmental outcomes (5-7). Earlier studies have consistently shown a relationship between increasing lead levels and decreasing IQ (7), with unexpectedly greatest negative impact on the IQ at lower ranges of elevated lead levels (8). More recent literature has suggested that even relatively low

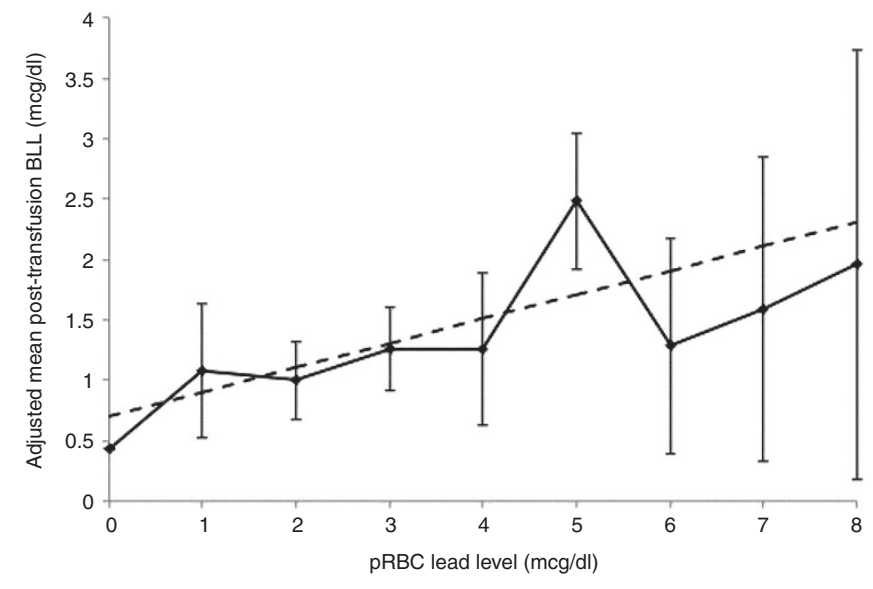

Figure 1. Adjusted mean (and $95 \% \mathrm{Cl}$ ) post-transfusion BLL by pRBC lead level. For each $1 \mathrm{mcg} / \mathrm{dl}$ increase in transfused pRBC lead level, infant posttransfusion BLL increased by $0.20 \mathrm{mcg} / \mathrm{dl}(95 \% \mathrm{Cl}: 0.07 \mathrm{mcg} / \mathrm{dl}, 0.33 \mathrm{mcg} /$ $\mathrm{dl}$; test of linear trend: $P=0.002$ ). The means are adjusted for gestational age, birth weight, and pretransfusion blood lead level.

levels can have a negative impact on cognition (8-11). This has prompted the CDC not only to further lower the reference ranges for toxic BLLs in children but also considering no BLL as safe and eliminate the term "blood lead level of concern"(2).

Prospective epidemiological studies have demonstrated that there may be a corelation between prenatal lead exposure and preterm delivery and low birth weight (17). There is also evidence that increased cord blood sample ( $>10 \mathrm{pg} / \mathrm{dl})$ may be associated with lower scores on Bayley Scales of Infant Development and continued deficits are seen for children with continued postnatal lead exposure along with an impact of socioeconomic factors $(18,19)$. Little to no information is available for the burden and impact of lead exposure in preterm infants. However, given that preterm brain is the most rapidly developing highly vulnerable organ as compared to term infants and children and 


\section{Articles | zubairietal.}

thus is probably more susceptible to toxic effects of lead and any lead exposure may be detrimental (1).

Lead neurotoxicity is thought to occur due to its effects at the cellular level, particularly affecting several parts of neurite growth in the developing brain. Schneider et al. (12) studied the effects of low-level lead exposure on survival and neurite length of rat E15 primary ventral mesencephalic dopaminergic neurons. They demonstrated that very low concentrations of lead, well below the level necessary to adversely affect neuronal survival, can impact neurite growth. It is hypothesized that lead affects overall regional growth as well as neuron-specific differentiation and synaptogenesis, a process thought to be most sensitive to low lead levels as compared to transient higher lead levels (20). One of the hypotheses to explain the underlying mechanism for these changes includes interference in the establishment of cell to cell connections with even transient lead exposure. In rats, a lead-specific binding protein typically transports zinc but, in the presence of lead, transports the lead into specific brain regions where protein is synthesized (21).

Potential sources of exposure for preterm infants include prenatal trans placental transmission, breast milk (for breastfed infants), and packed RBC transfusions. The American Congress of Obstetricians and Gynecologists therefore has clear guidelines for lead screening during pregnancy and lactation (22). For our cohort we noted that the baseline BLLs for majority of infants were below the $\mathrm{CDC}$ reference range for BLLs in children, thus eliminating trans-placental route as a potential exposure source. Lead exposure can occur through breast milk and has been linked to the duration of breastfeeding in population-based studies $(23,24)$. However, lead concentrations in breast milk are closer to plasma lead and much lower than blood lead and have been shown to be in relatively low concentrations despite long life time maternal exposure (25). In our cohort, we had a very high breastfeeding rate of $84 \%$ and this was similar for both the groups.

The pRBC aliquots are processed from adult donors and currently there are no procedures or policies for measuring the donor's BLL. The recent CDC weekly MMWR report noted that despite tremendous reductions in lead exposures over the past several decades in the United States, the National Institute for Occupational Safety and Health reports that some individuals continue to be exposed to lead at unacceptable levels $(26,27)$. A limitation of our study is that we only focused on the pRBCs and not the other transfusion products (platelets, fresh frozen plasma, and/or cryoprecipitate). This was done for two reasons-first we assumed that most of the lead load would be from the pRBCs (28-30) and second because currently the labs do not have a standardized method for testing lead levels in non-RBC blood products. Future studies should be designed to measure the lead levels in all blood products rather than solely focusing on the pRBCs so as to obtain even more accurate measure of exposure.

Our results are similar to those shown by previously by Bearer et al. (31) in their study of preterm infants, where they demonstrated that in a small sample of preterm population, multiple transfusions can result in post-transfusion lead levels of unacceptable values. In our study, we did note that this increase in lead levels was transient, as by the time of discharge there was no difference in the BLLs of the transfused versus the nontransfused group. This may simply be due to possible deposition of lead in soft tissues (brain, lung, liver, kidneys) as well as the mineralizing tissues (bone and teeth). Children retain more lead in soft tissues as compared to adults and there can be selective brain accumulation in the hippocampus. This raises the concerns that even transient exposure can significantly alter the neuronal growth potentially causing irreversible changes in the preterm brain. This makes it vital to eliminate any lead exposure at all by having BLL screening protocols in Transfusion Medicine and Blood Banks similar to the currently enforced infectious disease screenings. As more data become available, methodologies to chelate/remove lead from the donated blood products can be developed.

Infants and young children are more susceptible to the toxic effects of lead than adults, as well as are at an increased risk for lead accumulation in tissues. The amount of lead absorbed is inversely related to chronological age. Not only do infants have increased gastrointestinal absorption of lead as compared with adults (2) the absorbed lead accumulates in bone at higher levels than in adults potentially causing growth impairment and calcium deficiency $(31,32)$.The distribution of lead in preterm infants after absorption is not well studied with no data currently available; however, the assumption can be made that the distribution would be similar to that of adults and children $(33,34)$. Lead is known to be primarily excreted by the kidneys through urinary excretion with some biliary excretion in the stool. There is limited data about the daily excretion rates in preterm infants. It is known, that preterm infants metabolize lead differently and the majority of lead is not excreted in the urine at the same rates as older children and adults (35).

A big strength of our study was the direct linear relationship we found between any lead exposure from the transfusion aliquot with the subsequent post transfusion BLL in the transfused infant. The estimated $R^{2}$ values from the regression models reflect large effects, as defined by Cohen (36) (an $R^{2}$ value of 0.29 corresponds to a Cohen's $f^{2}$ value of 0.41 . The $R^{2}$ value of 0.37 corresponds to a Cohen's $f^{2}$ value of 0.59 ). This is not surprising recognizing that direct transfusion of $\mathrm{pRBC}$ with a given lead level will likely correspond with BLL obtained from the infant (37). One limitation to our study was that we did not measure urine lead levels and thus cannot conclude the amount of lead that may have been deposited in tissues versus excreted. This would help quantifying the true lead burden over time and should be addressed in future studies.

Further research is needed to focus on the impact of lead exposure on the developing brain and participants from this study are currently being followed at 18-24 mo of age to assess for any neurodevelopmental impairments.

\section{Conclusion}

Lead is a potent neurotoxicant with the developing brain at increased risk for adverse effects. Preterm infants routinely received blood transfusions in the neonatal intensive care unit setting and these can be potential sources of lead exposure. 
We found a statistically significant linear correlation between infant's post-transfusion lead levels and the lead level in the transfused aliquot and hypothesize that this exposure could lead to potential worse neurodevelopmental outcomes for these at risk infants.

\section{METHODS}

This was a prospective cohort study of infants $\leq 30 \mathrm{wk}$ completed gestational age who were admitted to the Davis Neonatal Intensive Care Unit, a Level III unit, at Baystate Children's Hospital, Springfield, MA between Jan 2011 to June 2012. We excluded infants who were $<23$ wk completed gestational age or infants with known chromosomal diseases with the potential for poor neurodevelopmental outcomes. Baseline data including gestational age, birth weight, gender, mode of delivery, 5-min APGAR score, hematocrit at admission and discharge, and any intraventricular hemorrhage, or periventricular leukomalacia were collected for each infant.

For each infant enrolled in the study, a baseline blood lead level (BLL) was obtained from cord blood at birth. If the cord blood was not available, a sample was collected at the first lab draw in the neonatal intensive care unit. For each transfusion, an infant received, a pretransfusion BLL and a level $6 \mathrm{~h}$ after the transfusion was completed (post-transfusion BLL) were obtained. This was done to allow time for equilibration of the transfused pRBCs. This was an empiric choice made as no data are currently available describing the optimal time for equilibration. We also obtained the lead level from the transfused donor aliquot of pRBCs. The lead load was then calculated for each transfusion using the following formula:

$\mathrm{Pb}$ transfused aliquot $(\mu \mathrm{g} / \mathrm{dl}) \times$ volume transfused $(\mathrm{ml})=\mathrm{Pb}$ load $(\mu \mathrm{g}) \times 100$

A discharge BLL was also obtained for each infant. Lead testing was sent to LabCorp, (Raritan, NJ) as per routine protocol and completed using inductively coupled plasma/mass spectrometry. The coefficient of variance for the test is $\pm 7 \%$ for the low levels and these are confirmed using test-retest procedure prior to reporting. We did not collect data for other blood product transfusions including platelets, fresh frozen plasma, and/or cryoprecipitate.

This study was approved by the Baystate Medical Center Institutional Review Board prior to conducting the study. A written informed consent was obtained from the parents or guardians of the enrolled infant prior to any study related blood sampling or data collection.

\section{Statistical Analysis}

Means and SDs, as well as medians and ranges, are reported for continuous measures. Frequencies are reported for categorical variables. BLLs were reported as integers with units of $\mathrm{mcg} / \mathrm{dl}$, except for values between 0 and $1 \mathrm{mcg} / \mathrm{dl}$ which were reported as $0.50 \mathrm{mcg} / \mathrm{dl}$. Univariable comparisons were conducted using unpaired $t$-tests for continuous variables, or the Wilcoxon rank-sum test, where indicated. Comparisons of transfusion groups on categorical variables were conducted with Fisher's exact test. To evaluate the influence of pBRC lead load on post-transfusion BLLs, a linear mixed regression model was then performed The multilevel regression model approach was used to account for repeated transfusions and assays within patients. Lead levels in the transfused RBC (i.e., pbRBC) were model two ways. First, $\mathrm{pbRBC}$ was modeled as a continuous variable to assess whether a linear trend was present. Second, pbRBC was modeled with indicators variable, which allowed the each level to be assessed independently of others. This second approach also enabled us to compute 95\% confidence intervals for each pbRBC level. These models controlled for pretransfusion BLLs, gestational age, and birth weight. An estimate of the multiple $R^{2}$ value for multilevel models is given by Snijders and Bosker and is interpreted as the proportional reduction in error for predicting an individual reading (36). All analyses were conducted in Stata (version 12.1, College Station, TX). Significance testing was conducted at a critical level of $5 \%$.

\section{ACKNOWLEDGMENTS}

The authors acknowledge the invaluable support provided by Lynn Eaton, MT (ASCP), CCRP, Research Coordinator, Departments of Diagnostic Services,
Pathology and Radiology at Baystate Medical Center for helping co-ordinate the sample preparations

\section{STATEMENT OF FINANCIAL SUPPORT}

This study was funded by the Baystate Health System Incubator Fund, Baystate Medical Center, Springfield, MA 01199.

Disclosure: Authors have no financial relationships or any conflict of interest disclosures relevant to this article to disclose.

\section{REFERENCES}

1. Sanders T, Liu Y, Buchner V, Tchounwou PB. Neurotoxic effects and biomarkers of lead exposure: a review. Rev Environ Health 2009;24:15-45.

2. Centers for Disease Control and Prevention. Response to Advisory Committee on Childhood Lead Poisoning Prevention Recommendations in "Low Level Lead Exposure Harms Children: A Renewed Call of Primary Prevention", 2012. http://www.cdc.gov/nceh/lead/ACCLPP/blood_lead_levels.htm.

3. American Academy of Pediatrics Policy Statement. Lead exposure in children: prevention, detection and management. Pediatrics 2005;116:1036-46.

4. Bellinger DC, Needleman HL. Intellectual impairment and blood lead levels. N Engl J Med 2003;349:500-2; author reply 500-2.

5. Needleman HL, Gatsonis CA. Low-level lead exposure and the IQ of children. A meta-analysis of modern studies. JAMA 1990;263:673-8.

6. Canfield RL, Henderson CR Jr, Cory-Slechta DA, Cox C, Jusko TA, Lanphear BP. Intellectual impairment in children with blood lead concentrations below 10 microg per deciliter. N Engl J Med 2003;348:1517-26.

7. Chandramouli K, Steer CD, Ellis M, Emond AM. Effects of early childhood lead exposure on academic performance and behaviour of school age children. Arch Dis Child 2009;94:844-8.

8. Lanphear BP, Hornung R, Khoury J, et al. Low-level environmental lead exposure and children's intellectual function: an international pooled analysis. Environ Health Perspect 2005;113:894-9.

9. Healey N, Jones-Otazo H, Walker M, et al. Toxicological Review and Recommended Toxicological Reference Values for Environmental Lead Exposure in Canada. Final Report: Prepared under contract to Health Canada. Prepared for the Contaminated Sites Division, Safe Environments Directorate, Healthy Environment and Consumer Safety Branch, Health Canada, Ottawa, 2010.

10. Carlisle JC, Dowling KC, Siegel DM, Alexeeff GV. A blood lead benchmark for assessing risks from childhood lead exposure. J Environ Sci Health A Tox Hazard Subst Environ Eng 2009;44:1200-8.

11. Wilhelm M, Heinzow B, Angerer J, Schulz C. Reassessment of critical lead effects by the German Human Biomonitoring Commission results in suspension of the human biomonitoring values (HBM I and HBM II) for lead in blood of children and adults. Int J Hyg Environ Health 2010;213:265-9.

12. Schneider JS, Huang FN, Vemuri MC. Effects of low-level lead exposure on cell survival and neurite length in primary mesencephalic cultures. Neurotoxicol Teratol 2003;25:555-9.

13. Luban NL. Management of anemia in the newborn. Early Hum Dev 2008;84:493-8.

14. Bell EF, Strauss RG, Widness JA, et al. Randomized trial of liberal versus restrictive guidelines for red blood cell transfusion in preterm infants. Pediatrics 2005;115:1685-91.

15. Kirpalani H, Whyte RK, Andersen C, et al. The Premature Infants in Need of Transfusion (PINT) study: a randomized, controlled trial of a restrictive (low) versus liberal (high) transfusion threshold for extremely low birth weight infants. J Pediatr 2006;149:301-7.

16. Bednarek FJ, Weisberger S, Richardson DK, Frantz ID 3rd, Shah B, Rubin LP. Variations in blood transfusions among newborn intensive care units. SNAP II Study Group. J Pediatr 1998;133:601-7.

17. Goyer RA. Results of lead research: prenatal exposure and neurological consequences. Environ Health Perspect 1996;104:1050-4.

18. Bellinger D, Leviton A, Waternaux C, Needleman H, Rabinowitz M. Longitudinal analyses of prenatal and postnatal lead exposure and early cognitive development. N Engl J Med 1987;316:1037-43.

19. McMichael AJ, Baghurst PA, Wigg NR, Vimpani GV, Robertson EF, Roberts RJ. Port Pirie Cohort Study: environmental exposure to lead and children's abilities at the age of four years. N Engl J Med 1988;319:468-75. 


\section{Articles | Zubairiet al.}

20. Lidsky TI, Schneider JS. Lead neurotoxicity in children: basic mechanisms and clinical correlates. Brain 2003;126(Pt 1):5-19.

21. Pennypacker KR, Xiao Y, Xu RH, Harry GJ. Lead-induced developmental changes in AP-1 DNA binding in rat brain. Int J Dev Neurosci 1997;15:321-8.

22. Lead screening during pregnancy and lactation. Committee Opinion No. 533. American College of Obstetricians and Gynecologists. Obstet Gynecol 2012;120:416-20.

23. Centers for Disease Control and Prevention. Guidelines for the identification and management of lead exposure in pregnant and lactating women, 2010. http://www.cdc.gov/nceh/lead/publications/leadandpregnancy2010.pdf.

24. Lozoff B, Jimenez E, Wolf AW, et al. Higher infant blood lead levels with longer duration of breastfeeding. J Pediatr 2009;155:663-7.

25. Ettinger AS, Téllez-Rojo MM, Amarasiriwardena C, et al. Levels of lead in breast milk and their relation to maternal blood and bone lead levels at one month postpartum. Environ Health Perspect 2004;112:926-31.

26. Shannon MW, Graef JW. Lead intoxication in infancy. Pediatrics 1992;89:87-90.

27. Very High Blood Lead Levels Among Adults - United States, 2002-2011. MMWR. http://www.cdc.gov/mmwr/preview/mmwrhtml/mm6247a6.htm. Accessed 24 March 2015.
28. Angle CR, McIntire MS. Red cell lead, whole blood lead, and red cell enzymes. Environ Health Perspect 1974;7:133-7.

29. Rosen JF, Trinidad EE. Significance of plasma lead levels in normal and lead-intoxicated children. Environ Health Perspect 1974;7:139-44.

30. Simons TJ. Lead transport and binding by human erythrocytes in vitro. Pflugers Arch 1993;423:307-13.

31. Bearer CF, O'Riordan MA, Powers R. Lead exposure from blood transfusion to premature infants. J Pediatr 2000;137:549-54.

32. Silbergeld EK. Mechanisms of lead neurotoxicity, or looking beyond the lamppost. FASEB J 1992;6:3201-6.

33. Agency for Toxic Substances and Disease Registry. Lead Toxicity. What is the Biological Fate of Lead. 2007. http://www.atsdr.cdc.gov/csem/csem. asp? $\mathrm{csem}=7 \& \mathrm{ppo}=9$.

34. Agency for Toxic Substances and Disease Registry. Toxicological profile for lead, 2007. http://www.atsdr.cdc.gov/toxprofiles/tp13.pdf.

35. Ziegler EE, Edwards BB, Jensen RL, Mahaffey KR, Fomon SJ. Absorption and retention of lead by infants. Pediatr Res 1978;12:29-34.

36. Cohen J. Statistical Power Analysis for the Behavioral Sciences. Lawrence Erlbaum Associates, Hillsdale, NJ, 1988.

37. Snijders TAB, Bosker RJ. Multilevel Analysis: An Introduction to Basic and Advanced Multilevel Modeling. Sage Publications, London, UK, 1999. 